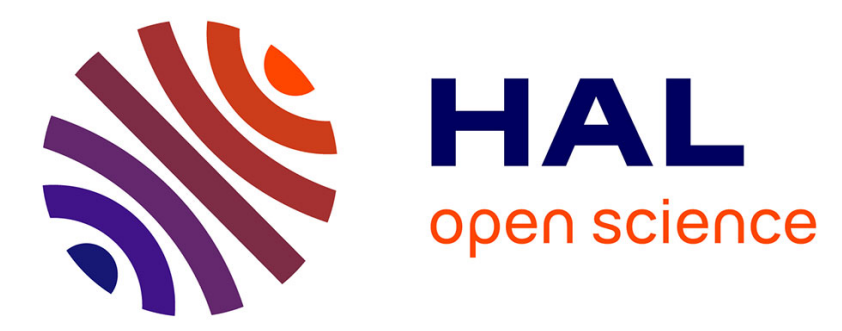

\title{
Rationale and geophysical evidence for quasi-geostrophic rapid dynamics within the Earth's outer core
}

Nicolas Gillet, Nathanaël Schaeffer, Dominique Jault

\section{To cite this version:}

Nicolas Gillet, Nathanaël Schaeffer, Dominique Jault. Rationale and geophysical evidence for quasigeostrophic rapid dynamics within the Earth's outer core. Physics of the Earth and Planetary Interiors, 2011, 202-203 (3-4), pp.78-88. 10.1016/j.pepi.2012.03.006 . insu-00561400

\section{HAL Id: insu-00561400 \\ https://hal-insu.archives-ouvertes.fr/insu-00561400}

Submitted on 1 Feb 2011

HAL is a multi-disciplinary open access archive for the deposit and dissemination of scientific research documents, whether they are published or not. The documents may come from teaching and research institutions in France or abroad, or from public or private research centers.
L'archive ouverte pluridisciplinaire $\mathbf{H A L}$, est destinée au dépôt et à la diffusion de documents scientifiques de niveau recherche, publiés ou non, émanant des établissements d'enseignement et de recherche français ou étrangers, des laboratoires publics ou privés. 


\title{
Rationale and geophysical evidence for quasi-geostrophic rapid dynamics within the Earth's outer core
}

\author{
Nicolas Gillet, Nathanaël Schaeffer, Dominique Jault \\ CNRS, Université Joseph Fourier, LGIT, BP 53, 38041 Grenoble Cedex 9
}

\begin{abstract}
We present arguments supporting the hypothesis that the flow in the Earth's core, for the time scales of the historical secular variation, is well described by a quasi-geostrophic (QG) model, almost invariant along the rotation axis. A previous study showed that for axisymmetric motions, the dimensionless number appropriate to compare magnetic and rotation forces is independent of magnetic diffusivity, increases with decreasing length scales, and takes value much smaller than unity for lengths about $10^{6} \mathrm{~m}$. Here, we extend this result to non-axisymmetric motions, and give a criterion for QG to hold based on length scale, rotation rate and magnetic field intensity. The numerical simulations exhibit a columnar behaviour at parameters representative of the Earth's core, supporting the quasi-geostrophic hypothesis for fast, large length-scale motions. In addition, we present the results of several inversions of the core flow from geomagnetic field models, showing that $(a)$ the energy of the motions symmetrical with respect to the equatorial plane represents about $80 \%$ of the total energy when no symmetry is assumed a priori; (b) for the same number of parameters, an equatorially symmetric $(\mathrm{QG})$ flow model explains more of the secular variation than a flow without specified geometry.
\end{abstract}

Keywords:

Earth's core, quasi-geostrophy, geomagnetic secular variation, core flow

\section{Background geophysical knowledge}

Combining the accuracy of satellite observations (Lesur et al., 2008; Olsen et al., 2009) and the quasi-geostrophic (QG) hypothesis for rapid core flows has made possible a much improved description of the core surface flows responsible for the secular variation (SV) of the Earth's magnetic field. Thus, Pais and Jault (2008) argued for the presence of a planetary scale, eccentric and anticyclonic gyre within the Earth's fluid core. Also, using an ensemble approach to account for the large scale (harmonic degree less than 13) secular variation produced from the invisible small scale radial magnetic field gave a realistic estimate of the variance of the core flow coefficients (Gillet et al., 2009). Moreover, using magnetic field data from the pre-satellite era together with the quasi-geostrophic hypothesis, gave indications on the temporal spectrum of the core flows (Gillet et al., 2010a). Finally, the QG hypothesis has 
been shown to be compatible with abrupt secular variation changes recorded at observatories (Gillet et al., 2009).

However, the QG hypothesis can be challenged. Recent studies about either the chemical interactions between the Earth's lower mantle and the fluid outer core (Buffett and Seagle, 2010), or the density of the topmost $300 \mathrm{~km}$ of the outer core inferred from observed seismic wave speeds (Helffrich and Kaneshima, 2010), have sparked renewed investigations of a stratified layer at the top of the core. If the stratification is strong enough, the motions in the layer should be decoupled from the interior flow with small horizontal scale (Takehiro and Lister, 2001). There is also some evidence that the heat flux at the core-mantle boundary is not laterally uniform (Lay et al., 2008). Then, a steady thermal wind could be driven at the top of the core (Amit et al., 2008) and may contribute to the geomagnetic secular variation. Finally, it has been suggested, on the basis of geodynamo numerical simulations, that magnetic diffusion plays an important role in the geomagnetic secular variation (Amit and Christensen, 2008). But it is in the context of negligible magnetic diffusion that the QG core flow hypothesis has been advocated (Jault, 2008).

Therefore, we find it important to introduce new empirical tests of the quasi-geostrophic core flow hypothesis. We follow different lines of argument. At the core surface, QG flows are particular tangentially geostrophic (TG, Le Mouël, 1984) core flows that are symmetrical with respect to the equatorial plane outside the trace $\mathcal{C}$, at the core surface, of the tangent cylinder (the cylinder tangent to the inner core and parallel to the rotation axis). Furthermore, QG flows do not cross the two curves $\mathcal{C}$. Hence, we can compare how well QG and TG core surface flows individually predict the observed secular variation for different epochs. From calculations of TG core flows, we can also simply investigate whether QG flows are predominant in the TG solutions. Furthermore, we can study whether the proportion of QG flows among the calculated TG flows increases with the accuracy of magnetic field data. For that latter purpose, we have found it necessary to use a magnetic field model which includes the last decades and accurately records the successive improvements of observatory series.

Lehnert (1954) introduced the number

$$
\lambda_{\ell}=\frac{B}{\ell \Omega \sqrt{\rho \mu_{0}}},
$$

which measures the ratio between the inertial wave period and the Alfvén wave period, and gives the magnitude of the magnetic force relative to the rotation force when magnetic diffusion is unimportant $\left(\boldsymbol{\Omega}=\Omega \boldsymbol{e}_{z}\right.$ is the rotation vector with $\boldsymbol{e}_{z}$ the unit vector along the rotation axis, $\ell$ is the characteristic length-scale, $B$ the magnetic field strength, $\rho$ the density and $\mu_{0}$ the free space magnetic permeability). The Lehnert number $\lambda_{\ell}$ is of the order of $510^{-4}$ in the Earth's core for $\ell \sim 10^{6} \mathrm{~m}$ and $B$ of the order of a few $\mathrm{mT}$ (Aubert et al., 2009; Gillet et al., 2009, 2010a). Jault (2008) gave different examples drawn from the literature of the occurrence of axially invariant flows in spherical shells for small values of $\lambda_{\ell}$. He also investigated numerically the fluid response, in a rapidly rotating spherical shell permeated by an axisymmetric (with respect to $\boldsymbol{e}_{z}$ ) magnetic field, to an impulsive rotation of the inner sphere. That transient response amounts to the propagation of two 
geostrophic shear layers from the tangent cylinder towards the equator of the outer sphere on the one hand and the rotation axis on the other hand. It contrasts with the zonal motions produced by a steady forcing, in which case the appropriate number to compare magnetic and rotation forces is the Elsasser number $\Lambda=\sigma B^{2} / \rho \Omega$ instead of the Lehnert number ( $\sigma$ is the electrical conductivity). For $\Lambda>O(1)$, the contours of equal differential rotation tend to follow the magnetic field lines of force, following Ferraro's law. In this paper, we generalize the study of Jault (2008) to non axisymmetric magnetic fields. We obtain again geostrophic shear layers propagating away from the tangent cylinder but also, superimposed on the geostrophic circulation, motions that are not axisymmetric. We find that they are mostly quasi-geostrophic for $\lambda_{\ell} \ll 1$.

After a brief introduction of the quasi-geostrophic approximation, we devote the following section to the numerical study, whereby we document the emergence of transient quasigeostrophic flows for $\lambda_{\ell} \ll 1$. We argue in the fourth section that our core flow inversions support the QG hypothesis. The description of the magnetic field model that we have used for this study is left for the Appendix C. In the last section, we discuss important open questions.

\section{The Quasi-geostrophic approximation}

The quasi-geostrophic approximation was first developed in the context of thin fluid geophysical envelopes (ocean, atmosphere, see e.g. Gill, 1982). It was later on adapted to thick spherical shells such as the Earth's outer core (Hide, 1966). QG models have given physical insight into the dynamics of rotating systems with varying height parallel to the axis or, in the case of thin shells, varying inclination of the rotation vector with respect to the local vertical. That latter ingredient is at the origin of phenomena such as Rossby waves, or generation of zonal motions through anisotropic turbulence (Smith and Waleffe, 1999; Read et al., 2004). It sets the QG model apart from a simple two-dimensional Navier-Stokes flow.

The quasi-geostrophic equations govern the evolution of the geostrophic velocity field $\boldsymbol{u}^{g}$ that satisfies the geostrophic equilibrium :

$$
2 \Omega \boldsymbol{e}_{z} \times \boldsymbol{u}^{g}=-\nabla \Pi^{g} \Rightarrow \boldsymbol{u}^{g}=\frac{1}{2 \Omega} \boldsymbol{e}_{z} \times \nabla \Pi^{g}(s, \phi) .
$$

$\left(\boldsymbol{e}_{s}, \boldsymbol{e}_{\phi}, \boldsymbol{e}_{z}\right)$ are unit vectors in the cylindrical coordinates $(s, \phi, z)$, and $\Pi^{g}$ is the reduced geostrophic pressure. The so-called barotropic flow $\boldsymbol{u}^{g}$ satisfies to the Proudman-Taylor constraint, $\frac{\partial \boldsymbol{u}^{g}}{\partial z}=\boldsymbol{O}$. The flow and pressure fields are completed by an ageostrophic (or baroclinic) component $\boldsymbol{u}^{a}$ and $\Pi^{a}$ :

$$
[\boldsymbol{u}, \Pi](s, \phi, z, t)=\left[\boldsymbol{u}^{g}, \Pi^{g}\right](s, \phi, t)+\left[\boldsymbol{u}^{a}, \Pi^{a}\right](s, \phi, z, t) .
$$

We find from (2) that $\boldsymbol{u}^{g}$ is divergence-free, which implies

$$
\nabla \cdot \boldsymbol{u}^{a}=0
$$


The QG model offers a way to investigate dynamics in a spherical shell, such as the Earth's core. It is formally justified for small Rossby numbers $R o=\frac{\mathcal{U}}{\Omega \ell}$, where $\mathcal{U}$ is the typical velocity. The Coriolis term associated with the ageostrophic flow $\boldsymbol{u}^{a}$ in the momentum equation cannot remain unbalanced. The $Q G$ approach consists in neglecting the ageostrophic contributions in the momentum equation that regroups the main perturbations to (2), except for the Coriolis and pressure terms:

$$
\frac{d \boldsymbol{u}^{g}}{d t}+2 \Omega \boldsymbol{e}_{z} \times \boldsymbol{u}^{a}=-\nabla \Pi^{a}+\frac{\boldsymbol{F}}{\rho}+\nu \nabla^{2} \boldsymbol{u}^{g} .
$$

$\boldsymbol{F}$ stands for the bulk forces and $\nu$ is the kinematic viscosity. Note that baroclinic instabilities are filtered in the above momentum equation. However, even in contexts where it does not formally apply ( $R o \sim 1$, presence of baroclinic waves), the QG approximation happens to give a useful framework, as shown by Williams et al. (2010).

Taking the curl of (5), in order to eliminate the pressure gradient, averaging along the $z$ component, and taking advantage of the incompressibility condition (4), permits to write an equation for the geostrophic pressure,

$$
\frac{d}{d t} \nabla^{2} \Pi^{g}+\frac{\beta}{s} \frac{\partial \Pi^{g}}{\partial \phi}=\frac{\Omega}{\rho H} \int_{-H}^{+H} \boldsymbol{e}_{z} \cdot(\nabla \times \boldsymbol{F}) d z+\nu\left(\nabla^{2}\right)^{2} \Pi^{g},
$$

for which we have used the boundary condition

$$
\boldsymbol{u} \cdot \boldsymbol{e}_{r \mid z= \pm H}=0 \Rightarrow \forall(s, \phi), u_{z \mid \pm H}= \pm \frac{d H}{d s} u_{s}^{g} .
$$

$H(s)=\sqrt{r_{o}^{2}-s^{2}}$ is the half-height of a fluid column, $\left(\boldsymbol{e}_{r}, \boldsymbol{e}_{\theta}, \boldsymbol{e}_{\phi}\right)$ are unit vectors in spherical coordinates $(r, \theta, \phi)$, and $r_{o}$ is the outer core radius. The slope of the container, which enters the expression for the parameter $\beta(s)=\frac{2 \Omega}{H} \frac{d H}{d s}$, is at the origin of Rossby waves.

Equation (6) must be coupled to an equation for the source term (density anomaly for buoyancy, magnetic field for the Lorentz force). Some variants of the QG set of equations for magnetized fluids, in which variations of the magnetic field with respect to $z$ are ignored, have been put forward (Hide, 1966; Busse and Finocchi, 1993; Diamond et al., 2007). They formed the basis of numerical models of the outer core rapid dynamics (Hide, 1966) and of the solar tachocline (Tobias et al., 2007). Canet et al. (2009) relied on the $z$-averaging of the Lorentz forces, as Gillet et al. (2007) before them, in order to write modified QG equations valid for a more general magnetic field in a spherical shell. Their model requires $\lambda_{\ell} \ll 1$ and that the field is much weaker at the fluid boundaries than in the interior. It has not been studied numerically yet.

As derived above, the QG model also gives an insight on the ageostrophic flow: crossing (5) with $\boldsymbol{e}_{z}$ before taking the $z$-derivative and eliminating the pressure term, through another application of (5), gives

$$
\frac{\partial \boldsymbol{u}_{e}^{a}}{\partial z}=\frac{1}{2 \rho \Omega} \boldsymbol{e}_{z} \times\left[\nabla_{e} F_{z}-\frac{\partial \boldsymbol{F}_{e}}{\partial z}\right]
$$


This is the thermal or magnetic wind equation, depending on $\boldsymbol{F}$. In the presence of axial variations of the magnetic field, the flows, which participate to the magnetostrophic balance (Taylor, 1963; Fearn, 1998) between Coriolis and magnetic forces, also present variations with respect to the $z$ coordinate. Nonetheless, these can be considered as part of the QG model defined above. In the QG framework, the contribution of the magnetostrophic flows to $\partial \boldsymbol{u} / \partial t$ is omitted.

Neglecting the vertical shear in (8) implies that $u_{z}^{a}$ varies linearly with $z$, as a result of (4). Then using (7), we are able to describe the vertical flow not only at the boundaries, but also in the core interior:

$$
\forall(s, \phi, z), u_{z}^{a}(s, \phi, z)=\frac{z}{H} \frac{d H}{d s} u_{s}^{g}(s, \phi) .
$$

Here, we have considered a fluid element outside the cylindrical surface tangent to the inner core (see Pais and Jault (2008) for the QG equations valid inside the tangent cylinder). In some sense, by using (2) and (9) to describe the flow we focus on the rapidly changing barotropic motions.

Hence, from a kinematical point of view, a QG velocity field is characterized by $(i)$ equatorial components $u_{s}$ and $u_{\phi}$ independent of $z$, and $(i i)$ a vertical component $u_{z}$ that obeys equation (9). Pais and Jault (2008) (see their section 4.3) show that theses two conditions imply the tangential geostrophy constraint at the core-mantle boundary:

$$
\nabla_{H} \cdot(\boldsymbol{u} \cos \theta)=0
$$

At this point, we have enough information to continue the velocity fields $\boldsymbol{u}(\theta, \phi)$ calculated at the core surface into the core interior, provided $(i)$ that the surface velocity obeys (10), (ii) that it is symmetrical with respect to the equatorial plane outside the tangent cylinder, and $($ iii) that

$$
u_{\theta}\left(\arcsin \left(r_{i} / r_{o}\right), \phi\right)=0
$$

where $r_{i}$ is the inner core radius.

Schaeffer and Cardin (2005) proposed an alternative derivation of the QG approximation in a sphere, whereby the equatorial flow $\boldsymbol{u}_{e}$ is assumed to be $z$-invariant, the vertical flow $u_{z}$ is still deduced from $u_{s}$ using (9), but

$$
\nabla \cdot \boldsymbol{u}_{e}=-\frac{\partial u_{z}}{\partial z}
$$

whereas definition (2) yields $\nabla \cdot \boldsymbol{u}_{e}^{g}=0$ instead. At the core surface and outside the tangent cylinder, (12) and (9) imply the constraint $\nabla_{H} \cdot \boldsymbol{u}=2 \tan \theta u_{\theta}$, first derived by Amit and Olson (2004) as the "columnar flow" constraint (see their Appendix A). Pais and Jault (2008) remarked that (12) and (9) imply a different constraint (their expression A8) inside the tangent cylinder TC. Thus, a consistent implementation of (12) requires either two different descriptions of the velocity field, respectively, inside and outside TC or ignoring the solid inner core altogether. Outside the tangent cylinder, Pais and Jault (2008) have found no major difference between flow models derived using either (2) or (12). 


\section{Quasi-geostrophic transient motions}

We already know from the previous study of Jault (2008) that transient axisymmetric motions that arise in a sphere permeated by an axisymmetric magnetic field are geostrophic and thus remarkably invariant along the rotation axis, even though the imposed magnetic field is not. Here, we extend this result to non-axisymmetric flows and magnetic fields. All the numerical experiments involve an electrically conducting fluid that occupies a spherical shell that is rotating at angular velocity $\Omega$ and is immersed in an imposed steady magnetic field $\boldsymbol{B}_{0}$. From a state of rest in the rotating frame (neglecting a possible background flow $\boldsymbol{U}_{0}$ ), we then perform a small and short displacement of the conducting inner sphere around the axis of rotation. The angular velocity of the inner sphere reaches a maximum value $\Delta \Omega$ and slows down back to rest during a few global rotation periods $T_{\Omega}=2 \pi / \Omega$. In contrast with the previous study of Jault (2008), $\boldsymbol{B}_{0}$ is not purely axisymmetric, leading to non-axisymmetric fluid motions.

Our three-dimensional spherical code (using second order finite differences in radius, pseudo-spectral spherical harmonic expansion and an Adams-Bashforth scheme in time) performs the time-stepping of the momentum equation of the fluid and of the induction equation, both in the conducting solid inner body and in the fluid spherical shell. We use no-slip boundary conditions. The region outside the spherical shell is electrically insulating and the solid inner body has the same electrical conductivity as the liquid in the spherical cavity.

Choosing the outer core radius $r_{o}$ as the length-scale, $B_{0}=\max \left(\left|\boldsymbol{B}_{0}\right|\right)$ as the magnetic field unit, the Alfvén time $\tau_{a}=r_{o} \sqrt{\rho \mu_{0}} / B_{0}$ as the time-scale, and $B_{0}^{2} / \mu_{0}$ as the pressure unit, we obtain the following non-linear dimensionless equations for the perturbations of the fluid velocity field $\boldsymbol{u}$ and of the magnetic field $\boldsymbol{b}$ :

$$
\begin{aligned}
\frac{\mathrm{d} \boldsymbol{u}}{\mathrm{d} t}+\frac{2}{\lambda_{r_{o}}} \boldsymbol{e}_{z} \times \boldsymbol{u} & =-\nabla \Pi+\left(\boldsymbol{j}+\boldsymbol{J}_{0}\right) \times\left(\boldsymbol{b}+\boldsymbol{B}_{0}\right)-\boldsymbol{J}_{0} \times \boldsymbol{B}_{0}+\frac{P_{m}}{S} \nabla^{2} \boldsymbol{u} \\
\frac{\partial \boldsymbol{b}}{\partial t} & =\nabla \times\left[\left(\boldsymbol{u}+\boldsymbol{U}_{f}\right) \times\left(\boldsymbol{B}_{0}+\boldsymbol{b}\right)\right]+\frac{1}{S} \nabla^{2} \boldsymbol{b},
\end{aligned}
$$

where $\Pi$ is the reduced pressure, $\boldsymbol{j}=\nabla \times \boldsymbol{b}$ and $\boldsymbol{J}_{0}=\nabla \times \boldsymbol{B}_{0}$ are the electrical currents associated respectively with the induced and imposed magnetic field. The imposed velocity field of the solid inner sphere is $\boldsymbol{U}_{f}(t)=r \sin \theta \Delta \Omega \exp \left[-\left(t / T_{f}-3\right)^{2}\right] \boldsymbol{e}_{\phi}$ for $r<r_{i}$ with $T_{f}=5 T_{\Omega}$. Since the amplitude of $\Delta \Omega$ is tiny, the nonlinear inertial term $(\boldsymbol{u} \cdot \nabla) \boldsymbol{u}$ does not play any important role. Note also that the model does not include the Lorentz force $\boldsymbol{J}_{0} \times \boldsymbol{B}_{0}$ that drives the background flow $\boldsymbol{U}_{0}$ omitted in this study.

The Lundquist number $S=\tau_{\eta} / \tau_{a}=r_{o} B_{0} / \eta \sqrt{\rho \mu}$, ratio of the magnetic dissipation time $\tau_{\eta}=r_{o}^{2} / \eta$ to the Alfvén wave time-scale, is of the order of $10^{5}$ in the Earth's core, with $\eta$ the magnetic diffusivity. The magnetic Prandtl number $P_{m}=\nu / \eta$, which is the ratio of the viscous over magnetic dissipation times, is of the order of $10^{-5}$ in the Earth's core. To keep as close as possible to the Earth, we use an aspect ratio $r_{i} / r_{o}=0.35$. The magnetic field intensity is chosen such that $10^{-4} \leq \lambda \leq 10^{-3}$, the magnetic diffusivity such that $10^{3} \leq S \leq 10^{4}$ and $\Lambda=O(1)$. These three parameter values are chosen to be similar 

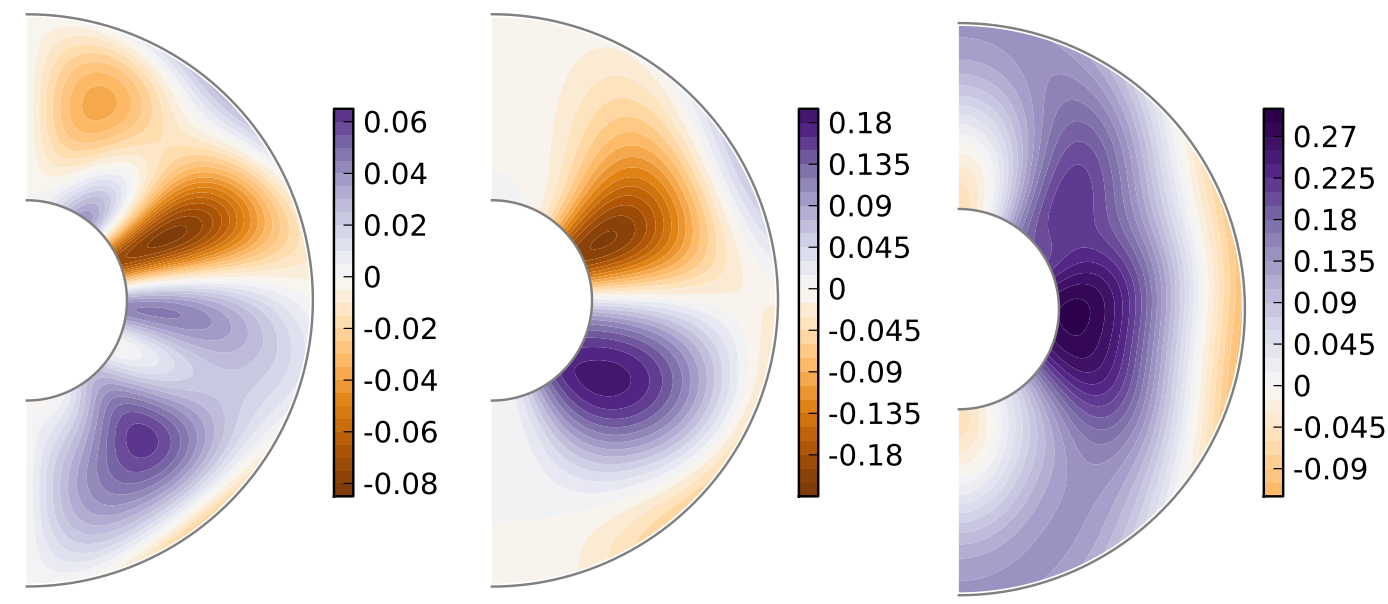

Figure 1: Meridional cross-section of the imposed magnetic field, in max $\left(\left|\boldsymbol{B}_{0}\right|\right)$ units. From left to right the cylindrical components $(s, \phi, z)$ of the magnetic field at $\phi=25^{\circ}$.

to those found in the Earth's core. As a consequence we reach magnetic Ekman number $E_{m}=\lambda / S=\eta / \Omega r_{o}^{2}<10^{-7}$. To keep the problem computationally tractable, we set $P m=1$. Table 1 gives the parameter values for the different numerical experiments.

The imposed magnetic field $\boldsymbol{B}_{0}$ is composed of an axisymmetric part (1/3 of the magnetic energy) and a non-axisymmetric part (2/3 of the magnetic energy) that has an azimuthal wave number $m=4$. There are no other symmetries imposed (the field contains both equatorially symmetric and antisymmetric components, see Appendix A for details). Meridional cross sections of the imposed field $\boldsymbol{B}_{0}$ are displayed in figure 1.

At the very beginning, just after the short displacement of the inner-core, most of the signal consists of inertial waves mainly propagating inside the tangent cylinder. Their effect is to quickly (in a few rotation periods $T_{\Omega}=2 \pi / \Omega$ ) form two strongly $z$-invariant, mostly axisymmetric and zonal jets, that propagate slowly as torsional Alfvén waves (Braginsky, 1970) away from the equator of the inner core. As already shown by Jault (2008), the axisymmetric component is geostrophic. We thus focus on the non-axisymmetric part of the flow, which arises because of the non-zonal component of the imposed magnetic field. It is illustrated with the equatorial cross-section shown in figure 2 for case A.

Figure 3 displays meridional cross-sections of the non-zonal flow for the cases $\mathrm{A}$ and $\mathrm{B}$, respectively 183 and $366 T_{\Omega}$ after the impulse. The flow contours are almost $z$-invariant: the Quasi-Geostrophic hypothesis holds well for transient non-axisymmetric motions, even though the Elsasser number $\Lambda$ reaches values up to 15 (see Table 1).

There are, however, localized motions which are not $z$-invariant. Figure 4 shows, for the cases $\mathrm{A}$ and $\mathrm{B}$, the cylindrical radial velocity at the same time but in another meridional cross-section where the magnetic field is locally about ten times stronger than in the cross- 


\begin{tabular}{|c|c|c|c|c|c|c|c|c|c|}
\hline case & $n_{\max }$ & $m_{\max }$ & $N_{r}$ & $E_{m}$ & $\Lambda$ & $\Lambda_{r m s}$ & $\lambda$ & $\lambda_{r m s}$ & $\mathrm{~S}$ \\
\hline $\mathrm{A}$ & 240 & 20 & 600 & $5.7 \times 10^{-8}$ & 15 & 1.5 & $9.1 \times 10^{-4}$ & $2.9 \times 10^{-4}$ & $1.6 \times 10^{4}$ \\
$\mathrm{~B}$ & 240 & 20 & 600 & $5.7 \times 10^{-8}$ & 2 & 0.2 & $3.4 \times 10^{-4}$ & $1.1 \times 10^{-4}$ & $6.0 \times 10^{3}$ \\
$\mathrm{C}$ & 240 & 20 & 1000 & $1.0 \times 10^{-8}$ & 12 & 1.2 & $3.4 \times 10^{-4}$ & $1.1 \times 10^{-4}$ & $3.4 \times 10^{4}$ \\
\hline
\end{tabular}

Table 1: Parameters for the numerical experiments. $\Lambda$ and $\lambda$ are based on the maximum value of the magnetic field in the fluid domain. The root-mean-squared values of these numbers is displayed for information. $n_{\max }$ and $m_{\max }$ are the truncation degree and order of the spherical harmonic expansion, $N_{r}$ is the number of radial grid points.
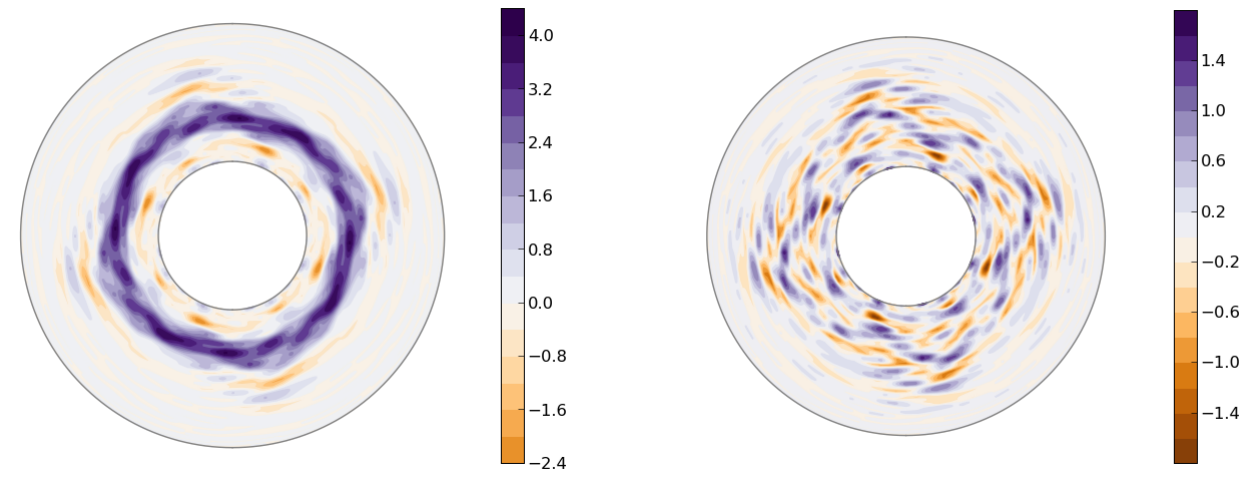

Figure 2: Equatorial cross-section of azimuthal component of the total (left) and non-axisymmetric (right) velocity field $u_{\phi}$ at $t=183 T_{\Omega}$ after the impulse (case A). The velocities are in units of $r_{o} \Delta \Omega / 1000$. 

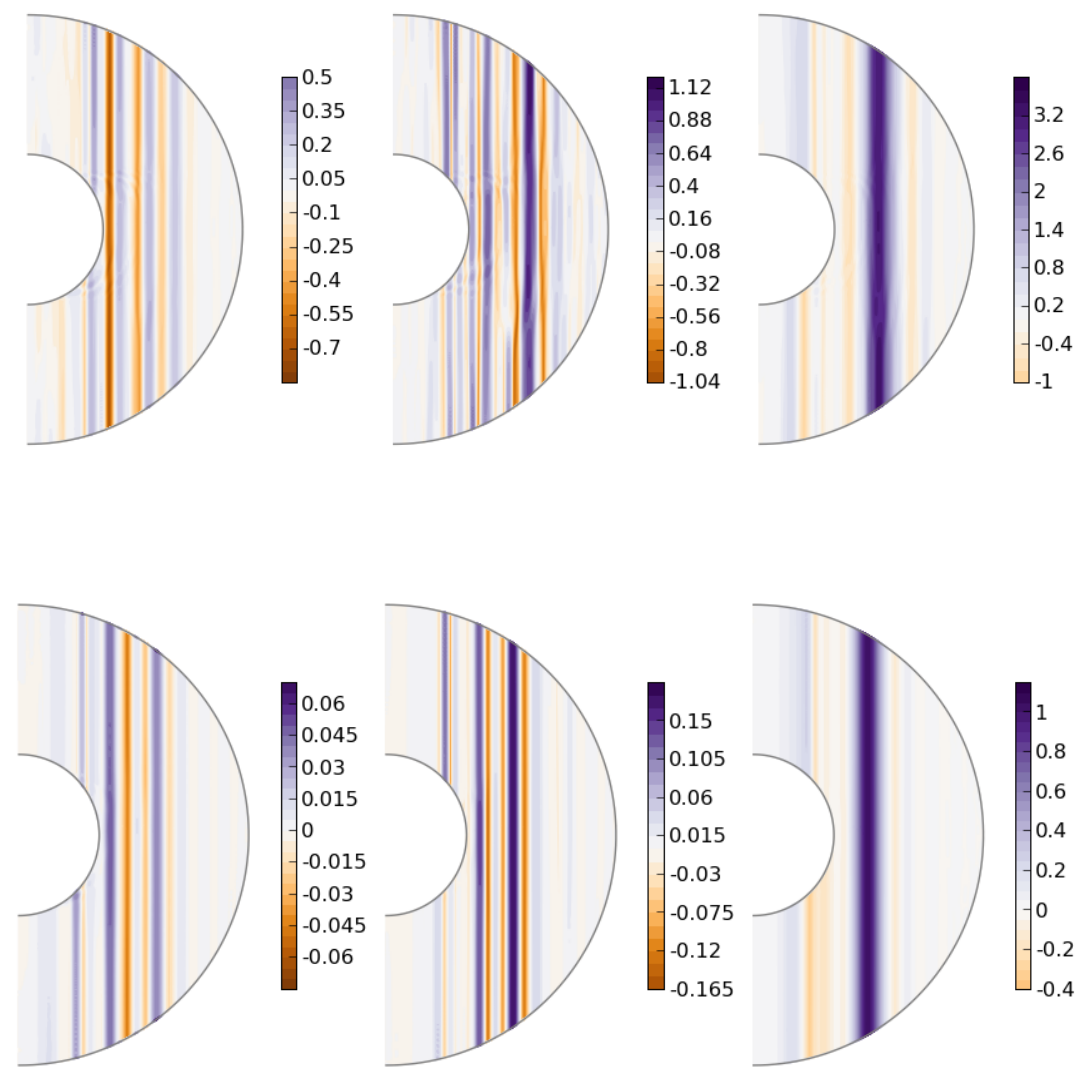

Figure 3: Meridional cross-sections of the velocity field. From left to right: the non-axisymmetric components of the velocity field $u_{s}$ (cylindrical radial) and $u_{\phi}$ (azimuthal) at $\phi=25^{\circ}$; and the axisymmetric component $u_{\phi}^{m=0}$. Velocities are in units of $r_{o} \Delta \Omega / 1000$. Top : case A at $t=183 T_{\Omega}$ after the impulse. Bottom : case $\mathrm{B}$ at $t=366 T_{\Omega}$ after the impulse. 

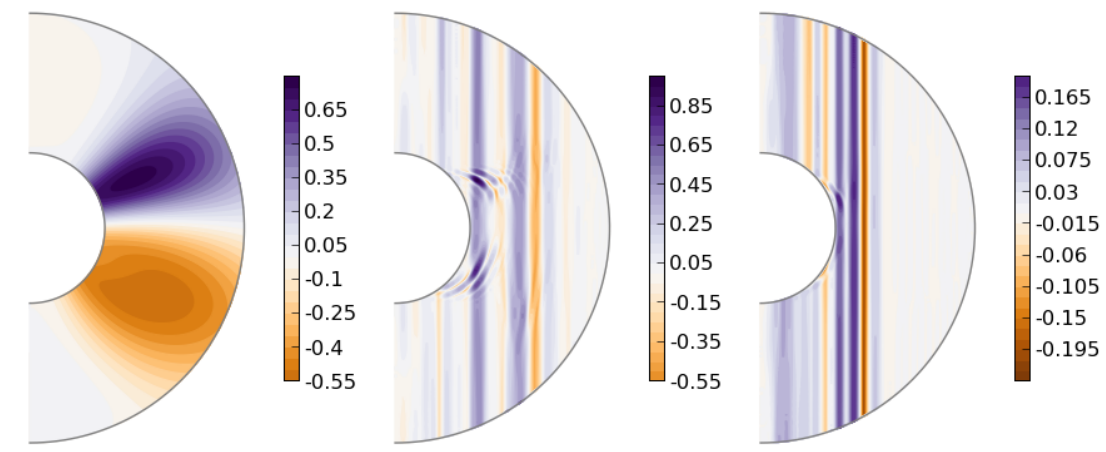

Figure 4: Meridional cross-section at $\phi=0^{\circ}$, where the magnetic field almost reaches its strongest intensity. Left: cylindrical radial component of the imposed magnetic field. Middle to right, cylindrical radial component $u_{s}$ for cases $\mathrm{A}$ and $\mathrm{B}$, respectively at $t=183 T_{\Omega}$ and $t=366 T_{\Omega}$ after the impulse. The magnetic field is in $\max \left(\left|\boldsymbol{B}_{0}\right|\right)$ units. Velocities are in units of $r_{o} \Delta \Omega / 1000$.

sections previously shown on figure 3. The QG hypothesis is locally violated in areas where the Elsasser number is about 10 and the local Lehnert number (based on the local length scale of the flow) is about 0.01 .

For the same meridional plane, a more quantitative description is given by the velocity profiles of figure 5 for the strongest magnetic field case (case A, $\phi=0$, where the Elsasser number based on the maximum $B_{s}$ in this plane would be about $\Lambda_{l o c}=6.3$ ). It seems that the large scales of the flow are more $z$-invariant than its small scales.

Figure 6 (case A) further contrasts the large scale and the small scale motions. The former are outstandingly $z$-invariant whereas the latter manifestly deviate from quasi-geostrophy. This result can be interpreted in terms of the value of the local Lehnert number (equation 1 ), which depends on the length scale. Quasi-geostrophy holds well, in figure 6, for length scales larger than 0.08 which means $\lambda_{\ell} \lesssim 0.01$. Of course, this also corresponds to an Elsasser number, and in order to discriminate between a limit given by the Elsasser number or by the Lehnert number, we use a filtering technique (see Appendix B) to determine the length scale above which the flow is QG. The results, reported in table 2, show that non-QG flow arise when the local Lehnert number $\lambda_{l o c} \lesssim 0.01$, with no influence of the Elsasser number. A similar estimation is provided by Jault (2008) in the axisymmetrical case. Applied to the Earth core, we would expect the liquid iron flow of length scale larger than $5 \times 10^{4} \mathrm{~m}$ to be strongly QG, corresponding to a spherical harmonic degree at the core surface of about 200.

We have thus exhibited flows that are both quasi-geostrophic and non axisymmetric, excited by an impulse on the rotation of the inner core. We find that the flows are even more QG when they are large length-scale as anticipated from the expression (1) for the Lehnert number. This also implies that the small scale, non-QG flows dissipate faster than the large scale QG flow. All our findings support the idea that the large scale flow in the Earth core should be quasi-geostrophic. In the following section, we search for empirical 


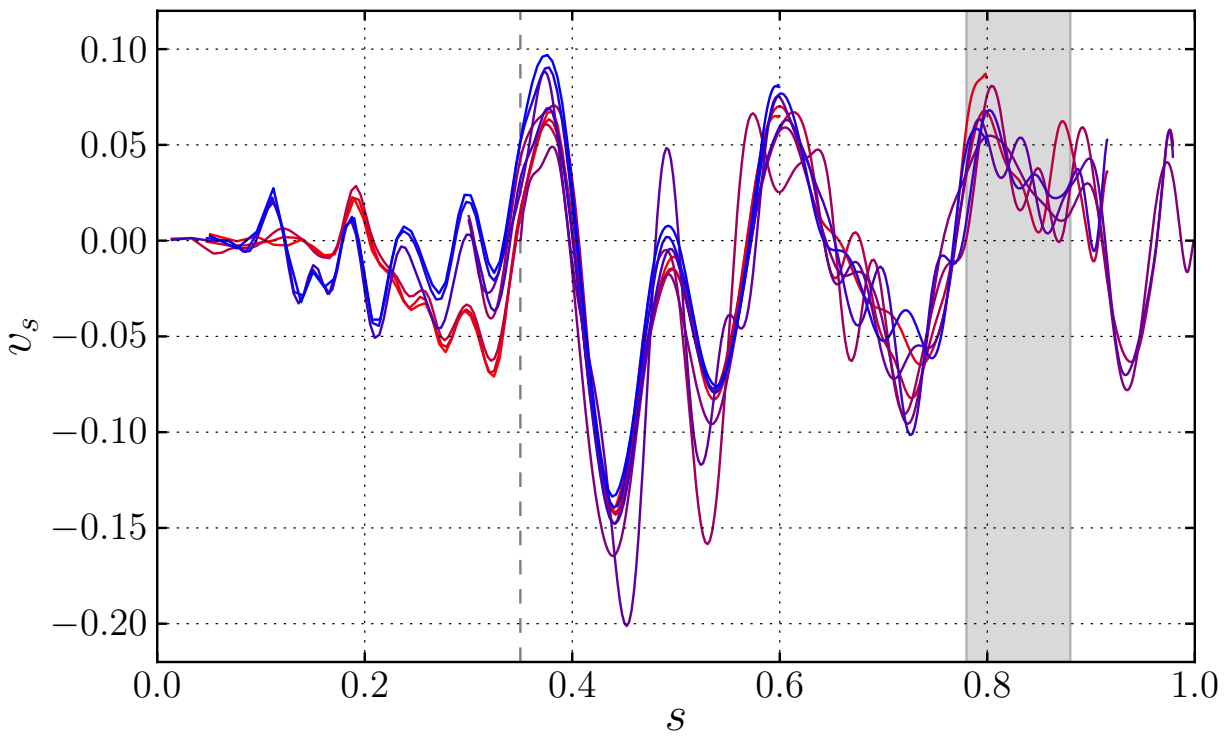

Figure 5: Velocity profiles $\left(u_{s}\right)$ as a function of cylindrical radius for different fixed height $(-0.8 \leq z \leq 0.8$ with steps of 0.2 , from blue to red) after $366 T_{\Omega}$, and at $\phi=0^{\circ}$ for case A. The shaded area indicates the location of the main torsional wave, and the velocities are in units of $r_{o} \Delta \Omega / 1000$.

\begin{tabular}{|c|c|c|c|c|c|}
\hline case & $E_{m}$ & $\Lambda$ & $\lambda$ & $\delta_{Q G}$ & $\lambda_{\delta}$ \\
\hline $\mathrm{A}$ & $5.7 \times 10^{-8}$ & 15 & $9.1 \times 10^{-4}$ & 0.10 & $9.1 \times 10^{-3}$ \\
$\mathrm{~B}$ & $5.7 \times 10^{-8}$ & 2 & $3.4 \times 10^{-4}$ & 0.04 & $8.5 \times 10^{-3}$ \\
$\mathrm{C}$ & $1.0 \times 10^{-8}$ & 12 & $3.4 \times 10^{-4}$ & 0.05 & $7.5 \times 10^{-3}$ \\
\hline
\end{tabular}

Table 2: For the three different parameter set, we have determined the length scale $\delta_{Q G}$ above which the flow is QG, and the corresponding Lehnert number (expression 1). Even though the Elsasser number spans almost one decade, $\lambda_{\delta}$ does not vary significantly. 

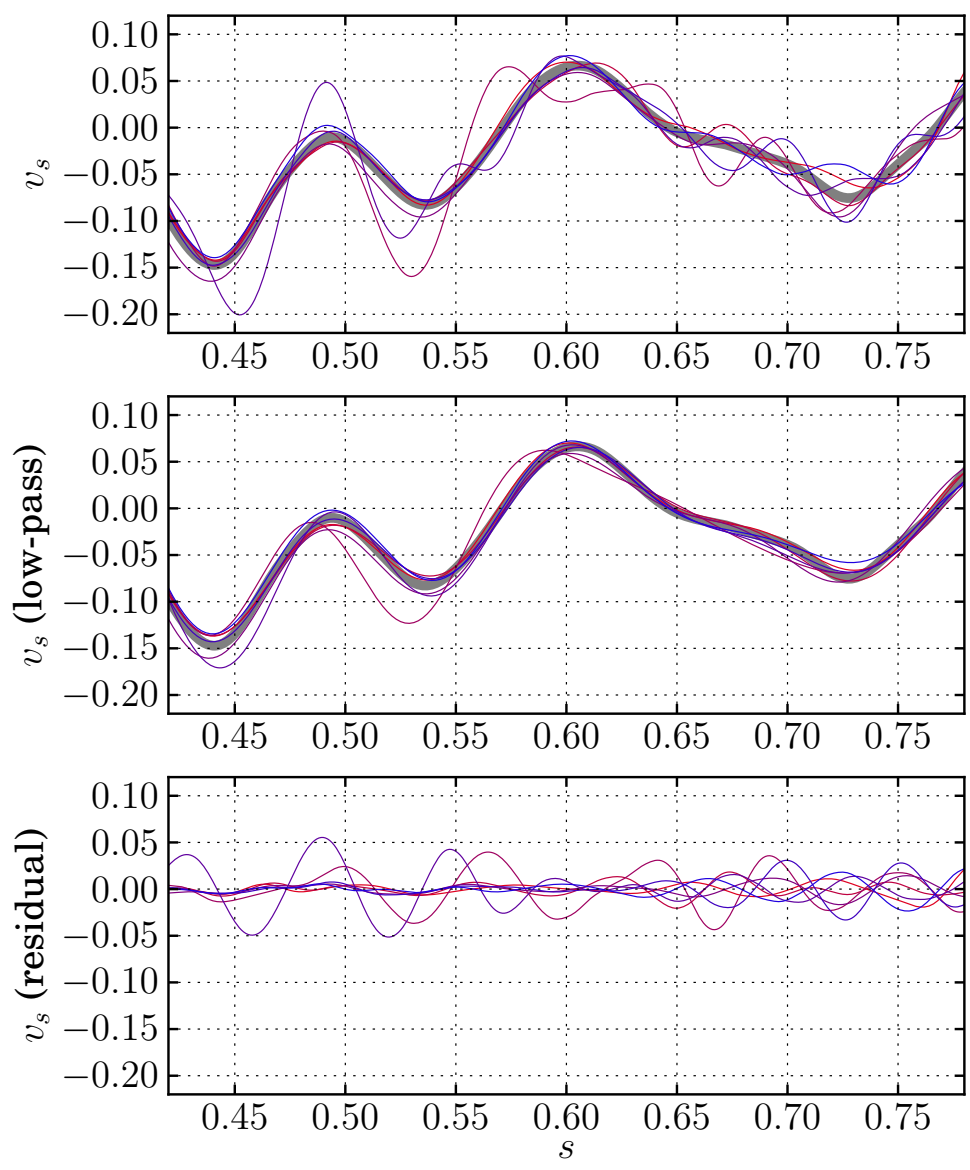

Figure 6: The data of figure 5 in the range $s \in[0.42,0.78]$ (top), after filtering out the scales smaller than 0.08 (middle), and the residual small scales (bottom). The bold grey curve is a $z$-average excluding the two most differing curves. Most of the departure from QG is contained in the small scales. 
evidence of quasi-geostrophy of the large scale core flows inferred from geomagnetic data.

\section{Tangentially- versus Quasi-geostrophic surface core flow models}

From the magnetic model obtained in Appendix C, we derive time-dependent core flow models spanning from year 1840.5 to 2008. The spherical harmonic representation $\mathbf{m}(t)$ of the Earth's magnetic field is considered as "data". It is used only up to degree $N_{b}=11$, since high degree coefficients are controlled by damping. The flow is calculated at the core surface. There, its toroidal and poloidal expansions (see e.g. Holme, 2007) $\mathbf{u}(t)=$ $\left\{t_{n m}^{s, c}(t), s_{n m}^{s, c}(t)\right\}_{n \in\left[1, N_{u}\right], m \in[0, n]}$, truncated at degree $N_{u}=22$, are expanded in time onto the same cubic B-spline basis as that used for the magnetic model. We follow an ensemble approach (Gillet et al., 2009), with ensemble size $K=20$, to account for the impact on the secular variation of unresolved main field coefficients of degree $n \in\left[N_{b}+1, \ldots, 3 N_{b}\right]$. We generate a set of random, time-correlated small-scale magnetic field models $\left\{\tilde{\mathbf{m}}^{k}(t)\right\}_{k=1, \ldots, K}$. For each realization in the ensemble, flow coefficients are linked to magnetic field coefficients through the forward problem

$$
\partial_{t} \mathbf{m}=\mathbf{A}\left(\mathbf{m}+\tilde{\mathbf{m}}^{k}\right) \mathbf{u}^{k}+\mathbf{e}^{k},
$$

where $\mathbf{e}^{k}$ is the secular variation errors vector. We use a strong form (Jackson, 1997; Gillet et al., 2009) to impose the tangentially geostrophic constraint (10) (Le Mouël, 1984). Extra topological constraints on the core surface flow derived from the QG approximation (equatorial symmetry outside the tangent cylinder plus zero cylindrical radial flow at the tangent cylinder) are applied with a weak form (Pais and Jault, 2008; Gillet et al., 2009). We then minimize a penalty function of the form

$$
J\left(\mathbf{u}^{k}\right)=\frac{1}{t_{e}-t_{s}} \int_{t_{s}}^{t_{e}}\left\{\left\|\partial_{t} \mathbf{m}-\mathrm{A}\left(\mathbf{m}+\tilde{\mathbf{m}}^{k}\right) \mathbf{u}^{k}\right\|_{\mathrm{C}_{\mathbf{m}}}^{2}+\alpha\left\|\mathbf{u}^{k}(t)\right\|_{\mathrm{R}}^{2}+\mu\left\|\mathbf{u}^{k}(t)\right\|_{\mathrm{Q}}^{2}\right\} d t
$$

with the generic notation $\|\mathbf{v}\|_{M}^{2}=\mathbf{v}^{T} \mathbf{M}^{-1} \mathbf{v}$. A constant noise level of $10(\mathrm{nT} / \mathrm{y})^{2}$ at the Earth surface has been considered to calculate the covariance matrix $C_{m}$. Diagonal elements of the regularization matrix $\mathrm{R}$ are proportional to $\frac{2 n+1}{[n(n+1)]^{2}}$, i.e. we use a penalization function intermediate between the kinetic energy norm and the "strong" norm (Holme, 2007). The TG and QG models analysed in the remaining of this section have been obtained with equation 16 and a coefficient $\alpha$ multiplying the regularizing norm set as $\alpha=10^{-5}$. We have previously noticed that the average of the ensemble of realizations, obtained using this norm, presents weak sensitivity to the damping parameter $\alpha$ over a wide range of $\alpha$ for degrees $n \leq 10$ (Gillet et al., 2009). TG flows are obtained with $\mu=0$, whereas for QG flows $\mu$ is set to a value large enough so that the solution does not vary when $\mu$ is further increased.

We first investigate the equatorial symmetry of the calculated TG flows. We note that the relative proportion of the flow component symmetrical about the equatorial plane (ES) increases with time as the data become more accurate (figure 7). We also plot the kinetic energy spectra for recent epochs (Figure 8). The energy of the flow component antisymmetrical 


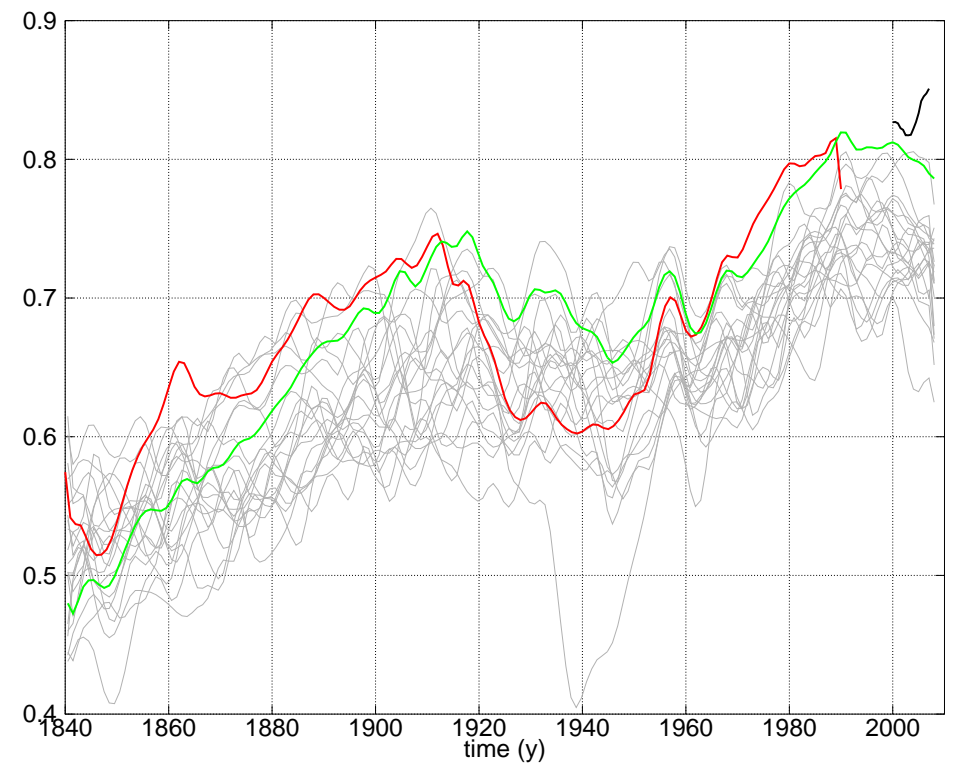

Figure 7: Time evolution of the fraction of ES kinetic energy for average TG flow models built from gufm1 (red), xCHAOS (black) and the model proposed in Appendix C (green). In grey: time evolution of the fraction of ES kinetic energy for all the realisations of flow obtained with our model.

about the equatorial plane (EA) is smaller than the energy associated with ES coefficients at low harmonic degrees $(n<10)$, as was already qualitatively remarked, 20 years ago, by Hulot et al. (1990) who wrote that their EA flows present no apparent global organization. We also find that most of EA coefficients are not resolved, since their dispersion is larger than their average value.

Then we compare the ability of TG and QG flows to account for the observed SV. We calculate surface core flows and then only keep the coefficients of degree $n \leq N$ of the resulting models. The part of the SV which is not explained by the flow models - the misfit - decreases with $N$. We thus obtain a relationship between the number $P$ of coefficients of the truncated flow model and the misfit (see Appendix D for the calculation of $P$ for TG and QG flow models). Figure 9 shows that for the same value of $P$, QG flows show a better fit to SV data than TG flows. This result is significant as the average flow models do not depend on the damping parameter for the resolved low degrees. As a consequence there is no difficulty for QG flows to predict sharp SV changes at observatory locations, as illustrated in Figure 10, and the delays observed for such events that depend on the observatory latitude or longitude (see e.g. Pinheiro and Jackson, 2008) cannot be used as an argument against the QG hypothesis.

We have already observed that the ES component of TG flows is enhanced when the quality of geomagnetic data, thus the accuracy of geomagnetic models, improves. Assuming this equatorial symmetry when calculating QG flows, we also find that the average of the ensemble of core flow models becomes more and more energetic as data quality increases, 


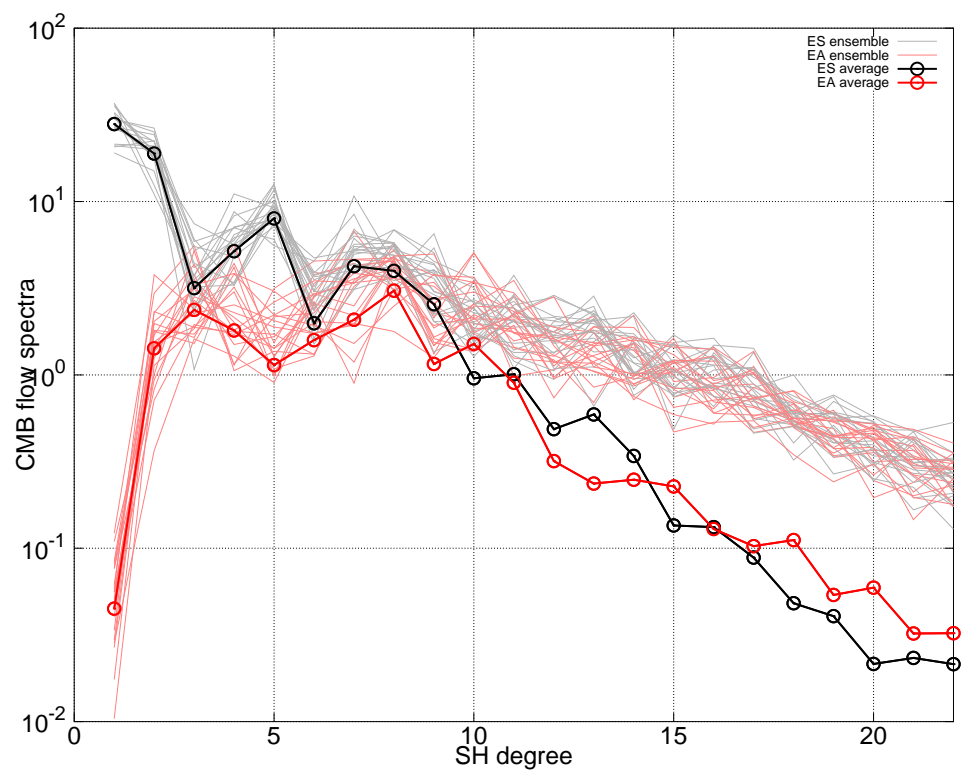

Figure 8: CMB flow spectra (time averaged over 1990-2000) for TG models: EA (red circles: average, light red: ensemble) and ES (black circles: average, grey: ensemble) flow components, in $(\mathrm{km} / \mathrm{yr})^{2}$.

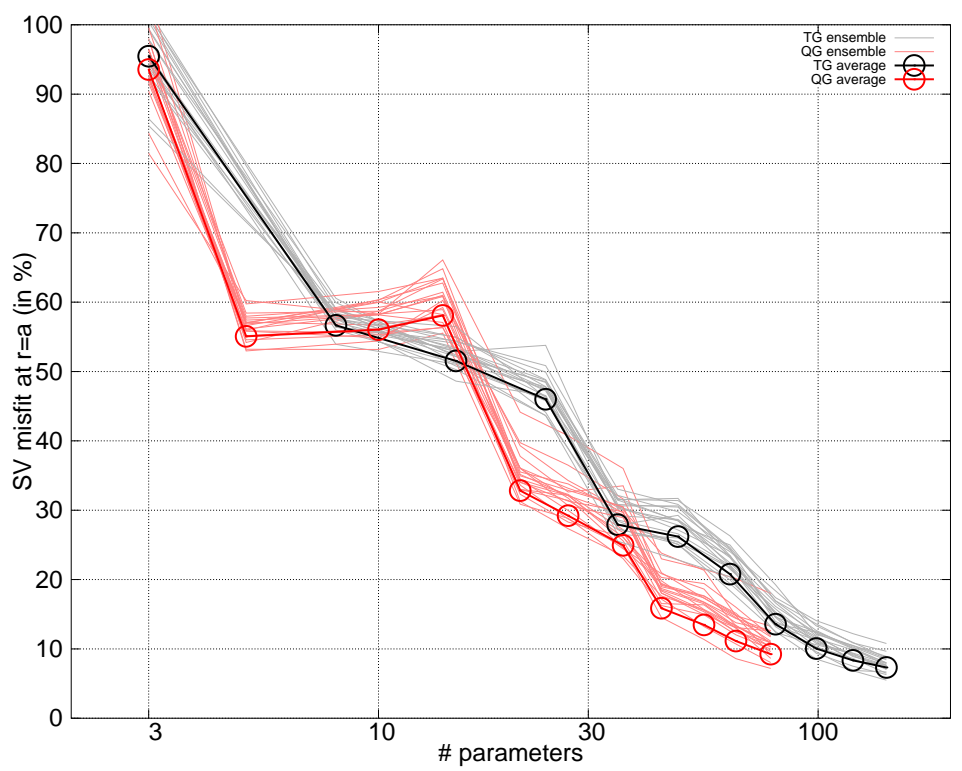

Figure 9: Misfit to the SV data (time averaged over 1990-2000) as a function of the number of parameters for TG (black circles: average, grey: ensemble) and QG (red circles: average, light red: ensemble) flow models truncated at different harmonic degrees. 

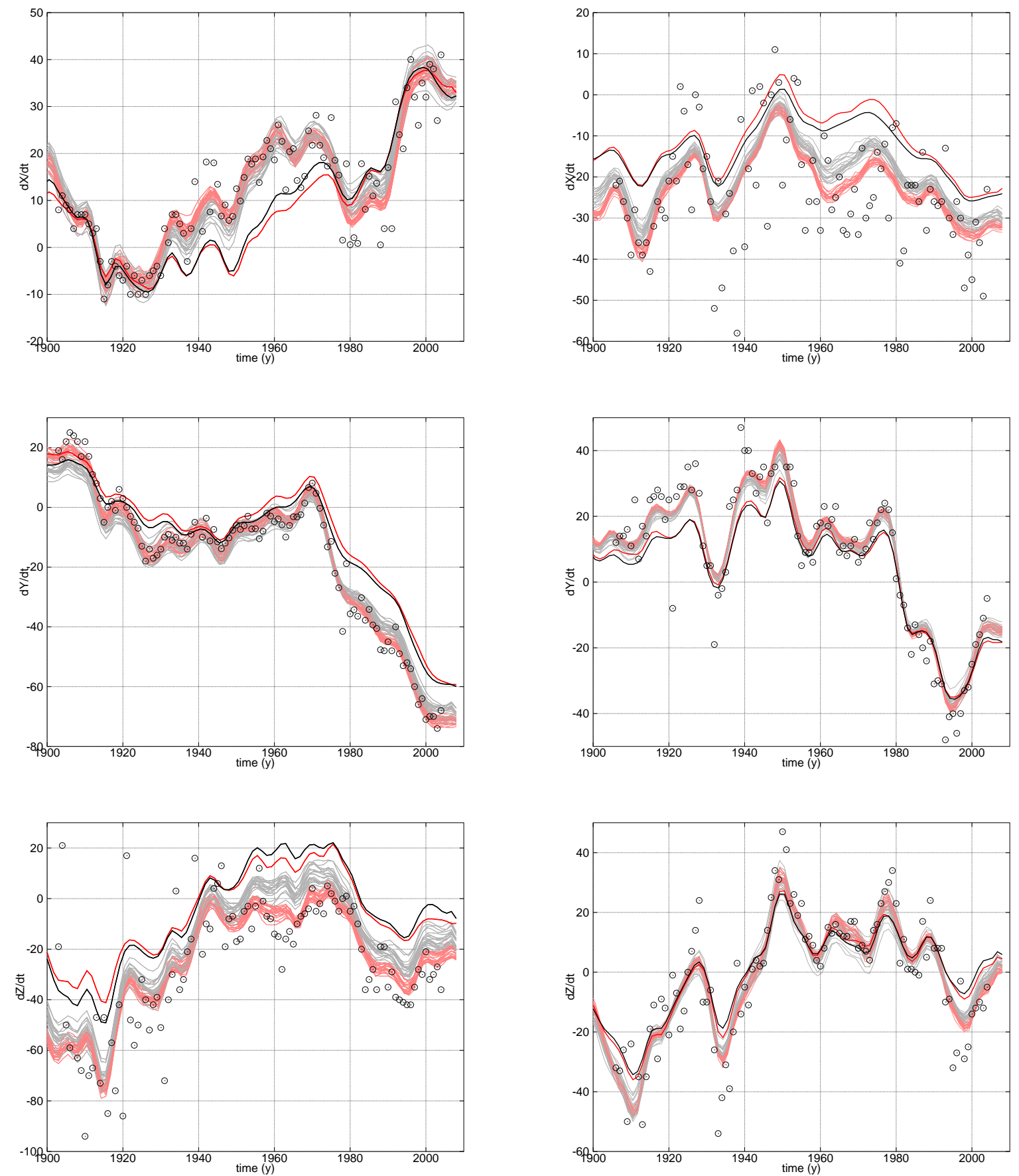

Figure 10: Comparison between SV predictions and first differences of annual means (black circles) recorded at the Sitka (Northern America, left) and Apia (South West Pacific, right) observatories in the X (top), Y (middle) and Z (bottom) directions. Full lines correspond to predictions from QG flow models obtained with damping parameters $\alpha=10^{-5}$ (ensemble: light red; average: red) and $\alpha=10^{-4}$ (ensemble: grey; average: black). 


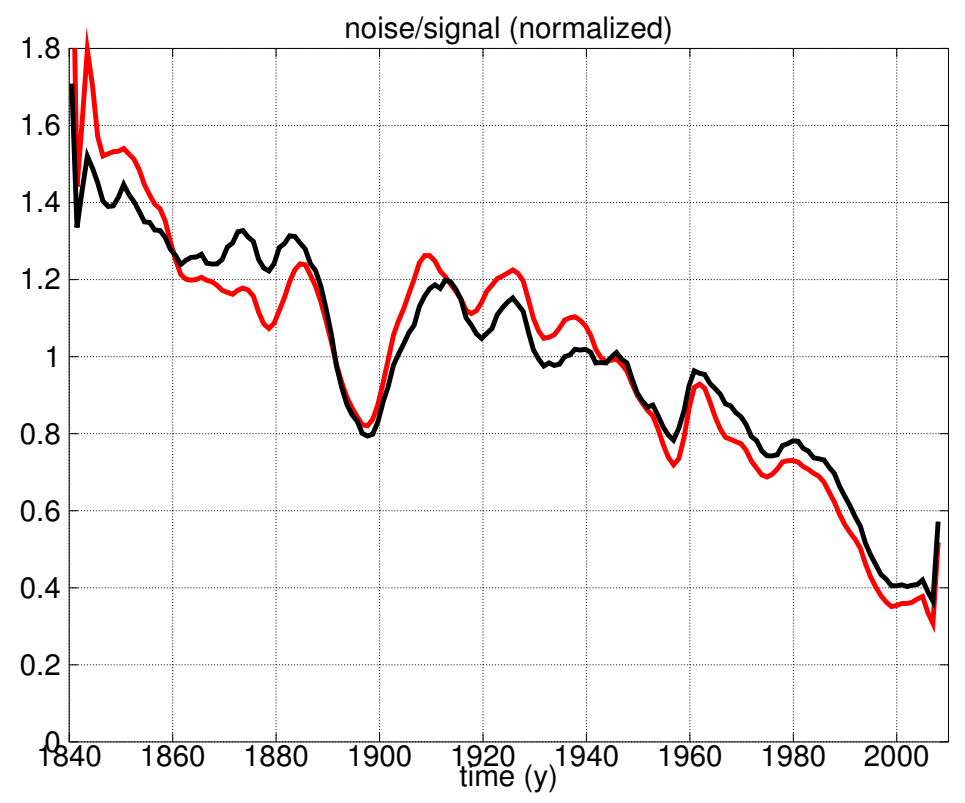

Figure 11: Time evolution of the ratio between the kinetic energy of the dispersion in the ensemble (variance) and the kinetic energy of the ensemble average, for damping parameters $\alpha=10^{-5}$ (black) and $\alpha=10^{-4}$ (red). Since our inversion technique only provides a relative estimate of the variance in the ensemble of core flow models (see Gillet et al., 2010a, supplementary information), we normalized both curves by their time average in order to obtain a meaningful comparison. 
leaving less and less flow coefficients unconstrained. This is illustrated in figure 11 that displays, as a function of time, the ratio between kinetic energies in the dispersion and the average of the ensemble, a kind of noise/signal ratio in the flow reconstruction. The decrease in the dispersion in the ensemble indicates that SV models are more easily predicted under the QG hypothesis when they are better constrained by geomagnetic data: when SV models are better constrained by geomagnetic data, less dispersion is observed in the ES component. We have checked that this observation does not depend on the choice of damping parameter $\alpha$. Gillet et al. (2009) had already pointed out this improving ability of QG flows at predicting SV data for the period 1960-2002, looking at the time evolution of the misfit between SV data and predictions. We extend here this analysis over the past 150 years. Our diagnostic is more rigorous than that used by Gillet et al. (2009), since the normalized quantity shown in figure 11 is less impacted by the 60 to 80 years apparent pulsation present in geomagnetic time series (Roberts et al., 2007).

\section{Geophysical \& methodological perspectives}

Our numerical models do suggest that large scale transient flows in the Earth's core are mostly quasi-geostrophic. We have not, however, accounted for the magnetostrophic velocity, which is formally part of the QG model (equation 8). Extensions of our numerical model are thus desirable. Before restoring the $\boldsymbol{J}_{0} \times \boldsymbol{B}_{0}$ term that we have neglected, we need to ensure that the prescribed magnetic fields used for the numerical experiment is in a Taylor state, namely that the circulation of the Lorentz force along geostrophic contours vanish when integrated over any geostrophic cylinder (Taylor, 1963; Livermore et al., 2010). Otherwise, fast geostrophic torsional waves will first develop. It would enable us to specify both the length scale and time scale at which the flow ceases to be axially invariant. We may also estimate the range of parameters for which the QG hypothesis holds by studying freely decaying turbulence or the response to a small scale turbulent forcing. As an example, one can wonder whether dipole changes on centennial time scales can be the result of QG dynamics (see the difficulty to reproduce the dipole trend from TG flow predictions according to Jackson, 1997).

For the purpose of this study, we have focused on the $z$-invariance of the motions that arise in our numerical experiment. However, as we pointed out in section 2, there are two different variations of the quasi-geostrophic model, leading to slightly different constraints on core-surface flows, especially in the equatorial region and in the vicinity of the tangent cylinder. Three-dimensional numerical simulations will be useful in pondering the validity of these constraints.

The ensemble technique of Gillet et al. (2009) was originally developed for satellite magnetic field models. In that context, there is a relatively well defined truncation level $N_{b} \simeq 13$ above which the magnetic field emanating from the core experiences too much geometric attenuation to be measurable at the Earth's surface in the presence of the crustal field. Here, we have only changed $N_{b}$ to $N_{b}=11$ to adapt the technique to a model derived from observatory and land survey data. That modification does not suffice to take the poor distribution of this type of data into account satisfactorily. Our suggestion is to forgo 
the customary but non-physical strong regularization of magnetic field models (as noted by Backus, 1988) and to generate the ensemble of magnetic field models used to calculate core flows from the covariance matrix of the field coefficients (following the stochastic approach of McLeod, 1996). That approach requires prior information on the magnetic field coefficients. A solution consists of extrapolating the empirical spatial spectra of the magnetic field and its secular variation calculated for low spherical harmonic degrees from recent satellite data to all degrees up to an arbitrarily large truncation degree. We plan then to reconsider the comparison between the observed and predicted length-of-day (LOD) changes over the 20th century. Indeed, the ensemble of core flows that are discussed in this paper tend to over predict the actual LOD changes. This is an indication that the largest scales of our time-varying flows have too high a velocity, probably as a consequence of the physically unjustified penalization of the small scale flows. As noted by Gillet et al. (2010b), the high degree SV coefficients of models derived from land data are less variable than the low degree coefficients which may unduly yield too variable large scale modelled core flows. Hopefully, introducing a priori information on the variability of the Gauss coefficients may counter that undesirable effect.

\section{Acknowledgements}

We are very grateful to Christopher Finlay and Andy Jackson for sharing with us the updated magnetic data sets used to build our magnetic model. We thank Phil Livermore, whose review helped improve the quality of the manuscript. This work has been supported by grants from the French Agence Nationale de la Recherche, Research programme VS-QG (grant number BLAN06-2.155316) and Centre National d'Études Spatiales. The simulations were run at the Service Commun de Calcul Intensif de l'Observatoire de Grenoble (SCCI).

\section{Appendix A. The imposed magnetic field}

Explicitly, we set $\boldsymbol{B}_{0}=\nabla \times \nabla \times(P \boldsymbol{r})$ with

$$
P=\left(j_{1}\left(\beta_{11} r\right)-0.3 j_{1}\left(\beta_{12} r\right)\right)\left(Y_{1}^{0}+\alpha \frac{1}{81} r^{2} Y_{9}^{4}\right)-0.2 j_{3}\left(\beta_{31} r\right)\left(Y_{3}^{0}+\alpha \frac{1}{144} r^{2} Y_{12}^{4}\right) .
$$

$(r, \theta, \phi)$ are the spherical coordinates. The $j_{n}$ 's are the spherical Bessel functions of the first kind, with $\beta_{n k}$ the $k$-th root of $j_{n-1} . Y_{n}^{m}(\theta, \phi)$ is the Schmidt semi-normalized spherical harmonic of degree $n$ and order $m$. Finally, $\alpha$ is a parameter that allows us to adjust the ratio of axisymmetric over non-axisymmetric components. In this study, $\alpha=1$ whilst $\alpha=0$ would reproduce the field of Jault (2008).

\section{Appendix B. Length scale separation in the numerical solution}

In our spherical code, the fields are defined by their spherical harmonic coefficients $A_{l}^{m}(r)$. Defining a spatial scale $\delta$ and the corresponding fractional spherical harmonic degree $l_{\delta}(r)=$ 
$\pi r / \delta$, we can compute

$$
\hat{A}_{l}^{m}(r)=e^{-\frac{l^{2}}{3 l_{\delta}^{2}(r)}}\left(\int e^{-3\left(r^{\prime}-r\right)^{2} / \delta^{2}} d r^{\prime}\right)^{-1} \int A_{l}^{m}\left(r^{\prime}\right) e^{-3\left(r^{\prime}-r\right)^{2} / \delta^{2}} d r^{\prime}
$$

which has the scales smaller than $\delta$ almost isotropically filtered out. $\delta_{Q G}$ is then the smallest $\delta$ for which the cylindrical components of the flow along the $\phi$ and $s$ direction do not show any significant variation in the $z$ direction.

\section{Appendix C. The geomagnetic model for 1840-2008 used in the study}

In order to document the impact, on core flow models reconstructed from geomagnetic field models, of increases in data quality that occured in the past century (use of proton magnetometers after 1960, digital acquisition introduced in the following decades), our study requires a continuous historical model covering the past century or so. This also makes possible to directly compare flow reconstructions estimated from models derived with or without satellite data of the past decade. Amongst the available models, gufm1 (Jackson et al., 2000) ends in 1990, whereas CM4 (Sabaka et al., 2004) only starts in 1960 and ends in 2002. We then decided to build an ad hoc new model covering 1840.5-2008 using a conventional approach. It starts in 1840 when intensity data become available (Gubbins et al., 2006; Finlay, 2008). We use extended data sets compared to that used for gufm1, adding in particular: first differences of observatory annual means up to 2006, and survey data up to 2005 from the BGS website (courtesy Susan MacMillan). The temporal evolution of the data frequency is displayed in Figure C.12.

As the method we follow is very similar to that used to build gufm1, we only recall here the main features. Under the insulating mantle hypothesis, the magnetic field derives from a potential. We perform a classical (Langel, 1987) spherical harmonics decomposition (degree $n$, order $m$ ) of the radial magnetic field $B_{r}$ at the core-mantle boundary, with truncation degree $N=14$. It defines the magnetic model $\mathbf{m}(t)=\left\{g_{n m}^{s, c}(t)\right\}_{n \in[1, N], m \in[0, n]}$. The time representation is similar to that of gufm1, using cubic B-spline basis (De Boor, 2001) with knots every 2.5 y regularly spanning $\left[t_{s}, t_{e}\right]=[1840.5,2008] . \mathbf{y}^{\mathbf{o}}=\left\{y_{i}\right\}_{i=1, \ldots, N^{\circ}}$ is the data vector, with $N^{o}$ the number of data. The geomagnetic forward problem is $\mathbf{y}^{\mathbf{o}}=\mathbf{H}(\mathbf{m})+\mathbf{e}^{\mathbf{o}}$, with $\mathbf{e}^{\mathbf{o}}$ the data error vector (with the associated error covariance matrix $\mathrm{C}$ ) and $\mathbf{H}$ the forward operator (see e.g. Gubbins and Roberts, 1983; Bloxham et al., 1989). We minimize a penalty function of the form

$$
J(\mathbf{m})=\chi^{2}+\frac{1}{t_{e}-t_{s}} \int_{t_{s}}^{t_{e}}\left\{\alpha_{S}\|\mathbf{m}(t)\|_{\mathrm{R}_{\mathrm{S}}}^{2}+\alpha_{T}\|\mathbf{m}(t)\|_{\mathrm{R}_{\mathrm{T}}}^{2}\right\} d t .
$$

$\chi^{2}=\left\|\mathbf{y}^{\mathbf{o}}-\mathbf{H}(\mathbf{m})\right\|_{\mathrm{C}}^{2}$ is the measure of the misfit to the data, with the notation $\|\mathbf{x}\|_{\mathrm{M}}^{2}=$ $\mathbf{x}^{T} \mathrm{M}^{-1} \mathbf{x} . \mathrm{R}_{\mathrm{S}}$ is the spatial damping matrix corresponding to the heating norm (Gubbins, 1975). $\mathrm{R}_{\mathrm{T}}$ is the temporal damping matrix penalizing for second time derivative (or secular acceleration) $\partial_{t}^{2} B_{r}$. 


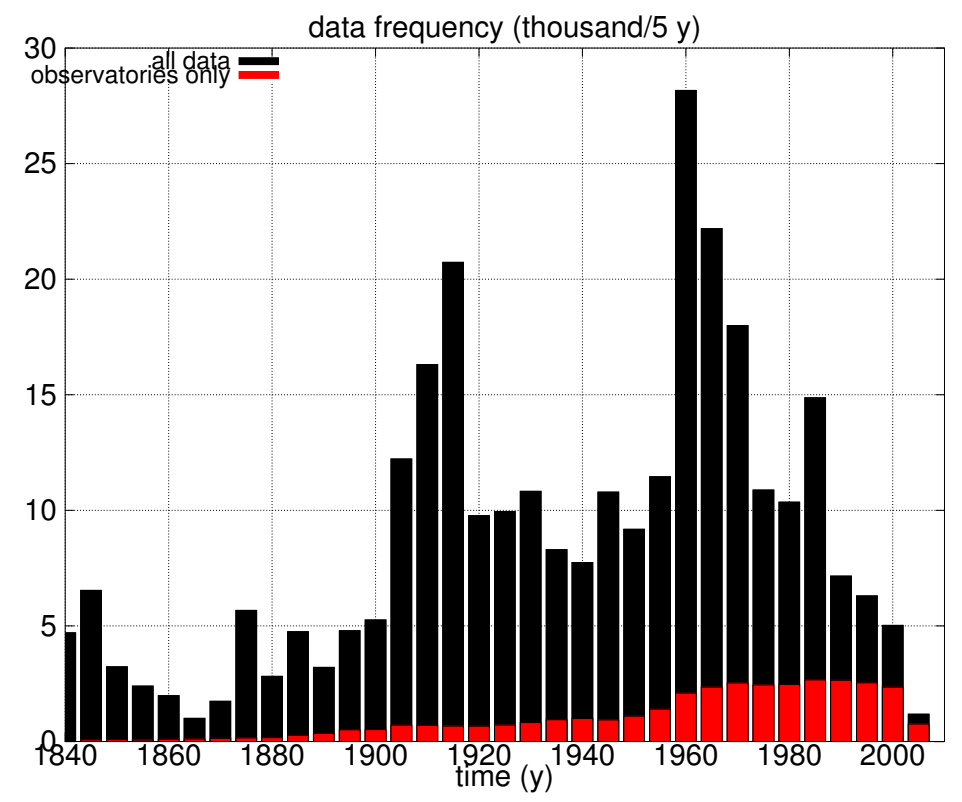

Figure C.12: Data frequency as a function of time, for all data (black), and observatory annual means only (red).

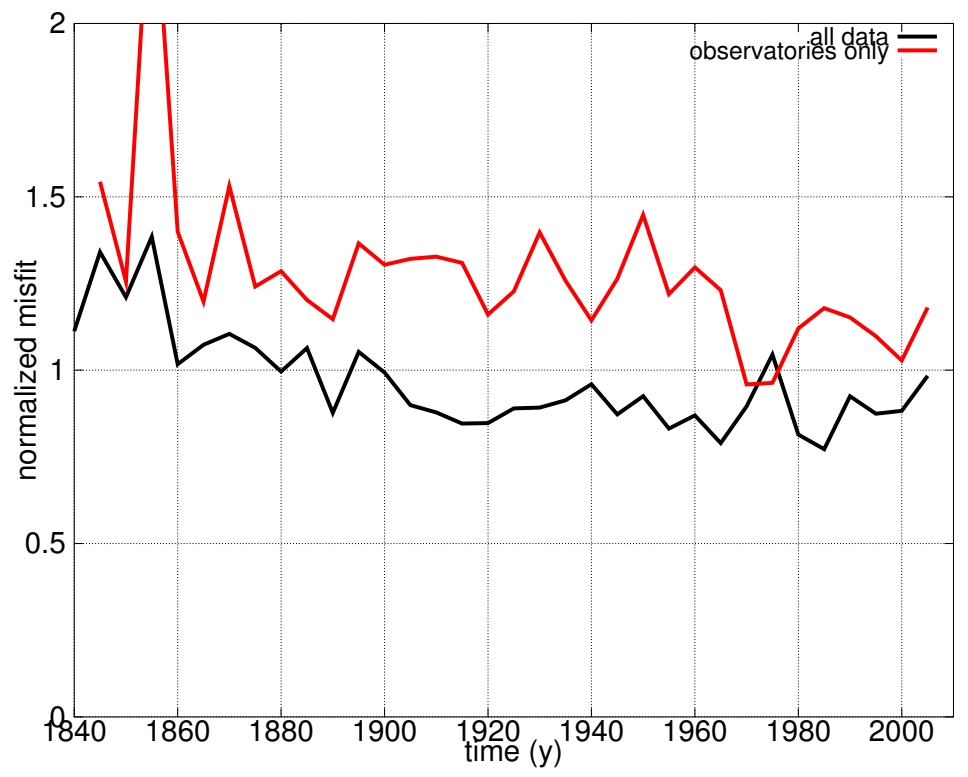

Figure C.13: Normalized misfit $\mathcal{M}$ as a function of time, for all data (black), and observatory annual means only (red). 


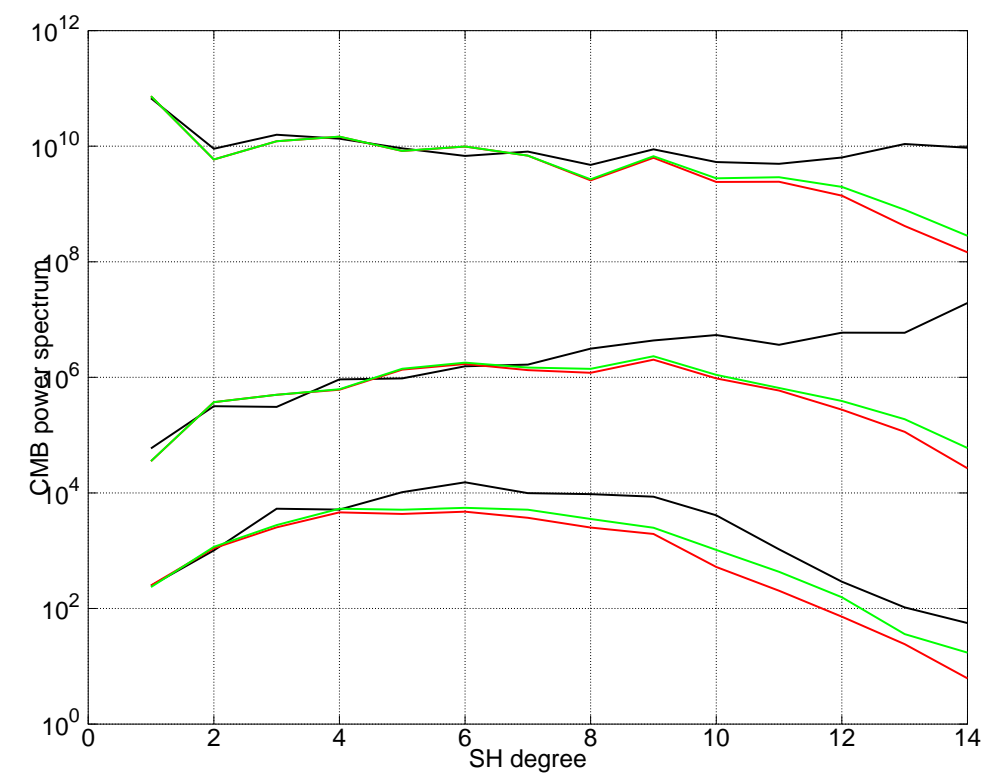

Figure C.14: From top to bottom: $\mathrm{MF}\left(\mathrm{nT}^{2}\right), \mathrm{SV}\left(\mathrm{nT}^{2} / \mathrm{y}^{2}\right)$ and $\mathrm{SA}$ (right, $\left.\mathrm{nT}^{2} / \mathrm{y}^{4}\right)$ spectra, time averaged respectively over 1900-1980 for the model derived for this work (green) and gufm1 (red), and over 2000-2006 for xCHAOS (black).

Our model is rather conservative: damping parameters $\left(\alpha_{S}, \alpha_{T}\right)=\left(10^{-10}, 5 \times 10^{-2}\right)$ have been chosen such that main field (MF), secular variation (SV) and secular acceleration (SA) norms and spectra are close to those obtained for gufm1 (see Figures C.14 and C.15). This choice of damping parameters provides a normalized misfit $\mathcal{M}=\sqrt{\chi^{2} / N^{o}}$ close to unity. Its temporal evolution is displayed in Figure C.13. The CMB power spectra and norms for the main field are defined as

$$
\begin{array}{r}
\mathcal{S}_{\mathrm{mf}}(n, t)=(n+1)\left(\frac{a}{c}\right)^{2 n+4} \sum_{m=0}^{N}\left[g_{n m}^{s}(t)^{2}+g_{n m}^{c}(t)^{2}\right], \\
\mathcal{N}_{\mathrm{mf}}(t)=\sqrt{4 \pi \sum_{n=1}^{N} \frac{n+1}{2 n+1} \mathcal{S}_{\mathrm{mf}}(n, t)},
\end{array}
$$

with similar expressions for the SV and SA.

\section{Appendix D. The number of parameters for TG and QG flow models}

There are $N_{u}\left(N_{u}+4\right)$ independent constraints for a TG model expanded up to degree $N_{u}$, so that remain $N_{u}^{2}$ degrees of freedom (Le Mouël et al., 1985; Jackson, 1997). However, $2 N_{u}$ constraints involve the poloidal coefficients $s_{N_{u}, m \neq 0}^{s, c}$, which are set to zero. These cannot be calculated with the geostrophic chain (see Backus and Le Mouël, 1986) as they require 

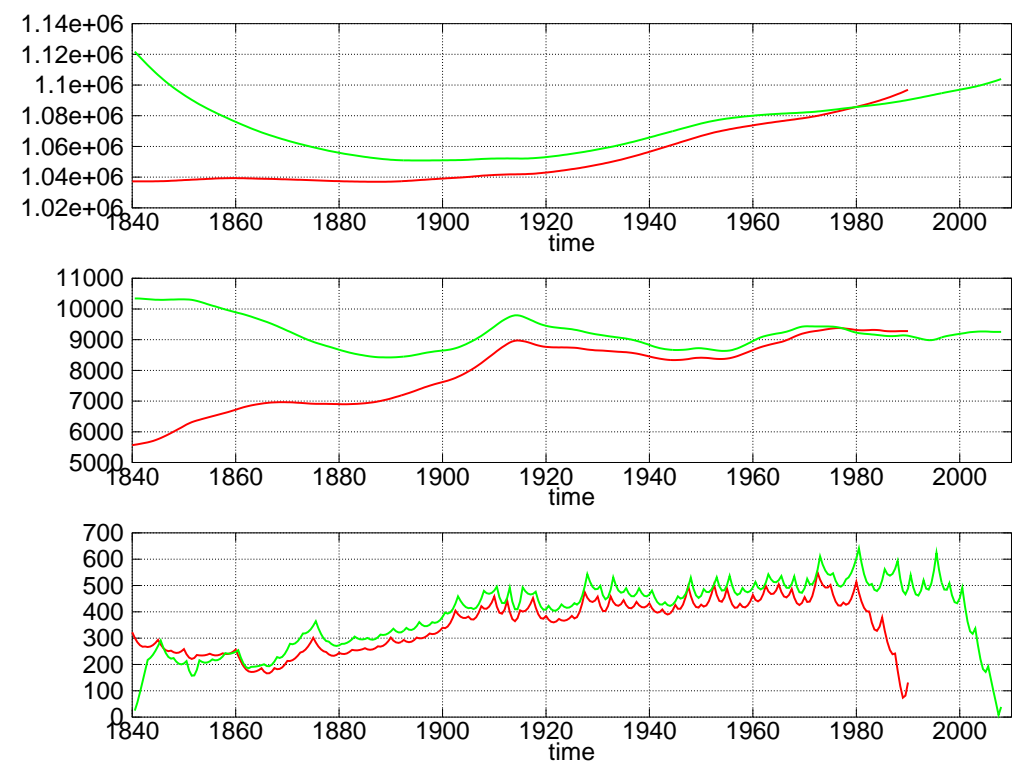

Figure C.15: From top to bottom: Magnetic Field (nT), Secular Variation (nT/y) and Secular Acceleration (right, $\mathrm{nT} / \mathrm{y}^{2}$ ) norms for the model used in this study (green) and gufm1 (red).

coefficients of degree $n>N_{u}$ that do not enter the flow model description. As a consequence, when truncating such a model at degree $N \leq N_{u}$, one actually finds that TG flow models contain $P=N(N+2)$ independent coefficients. We approximate $P$ for QG flows as the number of ES coefficients of a TG flow truncated at the degree $N: P=N(N+3) / 2$ for $N$ even and $P=N(N+3) / 2+1$ for $N$ odd. That estimate is not exact as we do not impose the equatorial symmetry constraint inside the tangent cylinder (which leaves more degrees of freedom, but concerns a small area), and we impose a constraint on the cylindrical radial flow at the tangent cylinder (which reduces the degrees of freedom).

Amit, H., Aubert, J., Hulot, G., Olson, P., 2008. A simple model for mantle-driven flow at the top of Earth's core. Earth Planets Space 60, 845-854.

Amit, H., Christensen, U., 2008. Accounting for magnetic diffusion in core flow inversions from geomagnetic secular variation. Geophysical Journal International 175 (3), 913-924.

Amit, H., Olson, P., 2004. Helical core flow from geomagnetic secular variation. Phys. Earth Planet. Inter. $147,1-25$.

Aubert, J., Labrosse, S., Poitou, C., 2009. Modelling the palaeo-evolution of the geodynamo. Geophys. J. Int. 179, 1414-1428, doi:10.1111/j.1365-246X.2009.04361.x.

Backus, G. E., 1988. Bayesian inference in geomagnetism. Geophysical Journal 92, 125-142.

Backus, G. E., Le Mouël, J.-L., 1986. The region on the core-mantle boundary where a geostrophic velocity field can be determined from frozen-flux magnetic data. Geophys. J. R. Astron. Soc. 85, 617-628.

Bloxham, J., Gubbins, D., Jackson, A., 1989. Geomagnetic secular variation. Phil. Trans. R. Soc. London A $329,415-502$.

Braginsky, S. I., 1970. Torsional magnetohydrodynamic vibrations in the Earth's core and variations in day length. Geomag. Aeron. 10, 1-8. 
Buffett, B., Seagle, C., 2010. Stratification of the top of the core due to chemical interactions with the mantle. Journal of Geophysical Research 115 (B4), B04407.

Busse, F., Finocchi, F., 1993. The onset of thermal convection in a rotating cylindrical annulus in the presence of a magnetic field. Physics of the Earth and Planetary Interiors 80 (1-2), 13-23.

Canet, E., Fournier, A., Jault, D., 2009. Forward and adjoint quasi-geostrophic models of the geomagnetic secular variation. J. Geophys. Res. 114, B11101, doi:10.1029/2008JB006189.

De Boor, C., 2001. A practical guide to splines. Applied Mathematical Sciences.

Diamond, P., Itoh, S., Itoh, K., Silvers, L., 2007. $\beta$-plane MHD turbulence and dissipation in the solar tachocline. In: Hughes, D., Rosner, R., Weiss, N. (Eds.), The Solar Tachocline. Cambridge University Press, pp. 213-239.

Fearn, D., 1998. Hydromagnetic flow in planetary cores. Reports on Progress in Physics 61, 175.

Finlay, C. C., 2008. Historical variations of the geomagnetic axial dipole. Phys. Earth Planet. Int. 70.

Gill, A. E., 1982. Atmosphere-ocean Dynamics. Academic Press.

Gillet, N., Brito, D., Jault, D., Nataf, H.-C., 2007. Experimental and numerical studies of magnetoconvection in a rapidly rotating spherical shell. J. Fluid Mech. 580, 123-143.

Gillet, N., Jault, D., Canet, E., Fournier, A., 2010a. Fast torsional waves and strong magnetic field within the Earth's core. Nature 465, 74-77.

Gillet, N., Lesur, V., Olsen, N., 2010b. Geomagnetic core field secular variation models. Space Sci. Rev. 155 (1), 129-145, doi:10.1007/s11214-009-9586-6.

Gillet, N., Pais, M. A., Jault, D., 2009. Ensemble inversion of time-dependent core flow models. Geochem. Geophys. Geosyst. 10 (6), Q06004, doi:10.1029/2008GC002290.

Gubbins, D., 1975. Can the Earth's magnetic field be sustained by core oscillation? Geophys. Res. Lett. 2, 409-412.

Gubbins, D., Jones, A. L., Finlay, C., 2006. Fall in Earth's magnetic field is erratic. Science 312, $900-903$.

Gubbins, D., Roberts, N., 1983. Use of the frozen flux approximation in the interpretation of archeomagnetic and paleomagnetic data. Geophys. J. R. Astr. Soc. 73, 675-687.

Helffrich, G., Kaneshima, S., 2010. Outer-core compositional stratification from observed core wave speed profiles. Nature, 807-810.

Hide, R., 1966. Free hydromagnetic oscillations of the Earth's core and the theory of geomagnetic secular variations. Phil. Trans. R. Soc. Lond. A 259, 615-646.

Holme, R., 2007. Large Scale Flow in the Core. In: Treatise in Geophysics, Core Dynamics, Vol. 8, pp107129. eds. P. Olson and G. Schubert.

Hulot, G., Le Mouël, J.-L., Jault, D., 1990. The flow at the core-mantle boundary: symmetry properties. J. Geomag. Geoelectr. 42, 857-874.

Jackson, A., 1997. Time-dependency of tangentially geostrophic core surface motions. Phys. Earth Planet. Inter. 103, 293-311.

Jackson, A., Jonkers, A. R. T., Walker, M. R., 2000. Four centuries of geomagnetic secular variation from historical records. Phil. Trans. R. Soc. Lond. A 358, 957-990.

Jault, D., 2008. Axial invariance of rapidly varying diffusionless motions in the Earth's core interior. Phys. Earth Planet. Int. 166, 67-76.

Langel, R. A., 1987. The main field. In: Geomagnetism. ed. J. A. Jacobs, vol. I, Academic Press, London.

Lay, T., Hernlund, J., Buffett, B. A., 2008. Core-mantle boundary heat flux. Nature geoscience 1, 25-32.

Le Mouël, J.-L., 1984. Outer core geostrophic flow and secular variation of Earth's geomagnetic field. Nature $311,734-735$.

Le Mouël, J.-L., Gire, C., Madden, T., 1985. Motions of the core surface in the geostrophic approximation. Phys. Earth Planet. Inter. 39, 270-287.

Lehnert, B., 1954. Magnetohydrodynamic waves under the action of the Coriolis force. Astrophysical Journal $119,647-654$.

Lesur, V., Wardinski, I., Rother, M., Mandea, M., 2008. GRIMM: the GFZ Reference Internal Magnetic Model based on vector satellite and observatory data. Geophys. J. Int. 173, 382-394.

Livermore, P., Ierley, G., Jackson, A., 2010. The construction of exact Taylor states. II: The influence of an 
inner core. Phys. Earth Planet. Int. 178, 16-26.

McLeod, M. G., 1996. Spatial and temporal power spectra of the geomagnetic field. J. Geophys. Res. 101, $2745-2763$.

Olsen, N., Mandea, M., Sabaka, T. J., Tøffner-Clausen, L., 2009. CHAOS-2 - a geomagnetic field model derived from one decade of continuous satellite data. Geophys. J. Int. 179, 1477-1487.

Pais, M. A., Jault, D., 2008. Quasi-geostrophic flows responsible for the secular variation of the Earth's magnetic field. Geophys. J. Int. 173, 421-443.

Pinheiro, K., Jackson, A., 2008. Can a 1-D mantle electrical conductivity model generate magnetic jerk differential time delays? Geophs. J. Int. 173, 781-792.

Read, P. L., Yamazaki, Y. H., Lewis, S. R., Williams, P. D., Miki-Yamazaki, K., Sommeria, J., Didelle, H., Fincham, A., 2004. Jupiter's and Saturn's convectively driven banded jets in the laboratory. Geophys. Res. Lett. 31, doi:10.1029/2004GL020106.

Roberts, P. H., Yu, Z. J., Russel, C. T., 2007. On the 60-year signal from the core. Geophys. Astrophys. Fluid Dyn. 101(1), 11-35.

Sabaka, T. J., Olsen, N., Purucker, M. E., 2004. Extending comprehensive models of the Earth's magnetic field with Oersted and CHAMP data. Geophys. J. Int. 159, 521-547.

Schaeffer, N., Cardin, P., 2005. Quasi-geostrophic model of the instabilities of the Stewartson layer in flat and depth varying containers. Physic of Fluids 17, 104111.

Smith, L. M., Waleffe, F., 1999. Transfer of energy to two-dimensional large scales in forced, rotating threedimensional turbulence. Phys. Fluids 11, 1608-1622.

Takehiro, S., Lister, J., 2001. Penetration of columnar convection into an outer stably stratified layer in rapidly rotating spherical fluid shells. Earth and Planetary Science Letters 187 (3-4), 357-366.

Taylor, J. B., 1963. The magnetohydrodynamics of a rotating fluid and the Earth's dynamo problem. Proc. R. Soc. London A 274, 274-283.

Tobias, S. M., Diamond, P. H., Hughes, D. W., 2007. $\beta$-plane magnetohydrodynamics turbulence in the solar tachocline. Astrophys. J. 667, 113-116.

Williams, P. D., Read, P. L., Haines, T. W. N., 2010. Testing the limits of quasi-geostrophic theory: application to observed laboratory flows outside the quasi-geostrophic regime. J. Fluid. Mech. 49, 187-203. 